\title{
Evaluation of the Performance of Small Diode Pumped UV Solid State (DPSS) Nd:YAG Lasers as New Radiation Sources for Atmospheric Pressure Laser Ionization Mass Spectrometry (APLI-MS)
}

\author{
Hendrik Kersten, Matthias Lorenz, Klaus J. Brockmann, Thorsten Benter
}

Department of Physical and Theoretical Chemistry, University of Wuppertal, Gauss Str. 20, 42119, Wuppertal, Germany

\begin{abstract}
The performance of a $\mathrm{KrF}^{*}$ bench top excimer laser and a compact diode pumped UV solid state (DPSS) Nd:YAG laser as photo-ionizing source in LC-APLI MS is compared. The commonly applied bench-top excimer laser, operating at $248 \mathrm{~nm}$, provides power densities of the order of low $\mathrm{MW} / \mathrm{cm}^{2}$ on an illuminated area of $0.5 \mathrm{~cm}^{2}(8 \mathrm{~mJ} /$ pulse, $5 \mathrm{~ns}$ pulse duration, beam waist area $0.5 \mathrm{~cm}^{2}, 3 \mathrm{MW} / \mathrm{cm}^{2}$ ). The DPSS laser, operating at $266 \mathrm{~nm}$, provides higher power densities, however, on a two orders of magnitude smaller illuminated area $\left(60 \mu \mathrm{J} /\right.$ pulse, $1 \mathrm{~ns}$ pulse duration, beam waist area $\left.2 \times 10^{-3} \mathrm{~cm}^{2}, 30 \mathrm{MW} / \mathrm{cm}^{2}\right)$. In a common LC-APLI MS setup with direct infusion of a $10 \mathrm{nM}$ pyrene solution, the DPSS laser yields a significantly smaller ion signal $(0.9 \%)$ and signal to noise ratio $(1.4 \%)$ compared with the excimer laser. With respect to the determined low detection limits (LODs) for PAHs of $0.1 \mathrm{fmol}$ using an excimer laser, LODs in DPSS laser LC-APLI MS in the low pmol regime are expected. The advantages of the DPSS laser with respect to applicability (size, cost, simplicity) may render this light source the preferred one for APLI applications not focusing on ultimately high sensitivities. Furthermore, the impact of adjustable ion source parameters on the performance of both laser systems is discussed in terms of the spatial sensitivity distribution described by the distribution of ion acceptance (DIA) measurements. Perspectives concerning the impact on future APLI-MS applications are given.
\end{abstract}

Key words: Atmospheric pressure laser ionization, APLI, Excimer laser, DPSS solid state laser, LC-MS, Distribution of ion acceptance, DIA

\section{Introduction}

A mong the variety of atmospheric pressure ionization (API) methods such as electrospray ionization (ESI), atmospheric pressure chemical ionization (APCI), and atmospheric pressure photo-ionization (APPI), atmospheric pressure laser ionization (APLI) is the youngest technique that has become commercially available [1]. APLI targets

Correspondence to: Hendrik Kersten; e-mail: hkersten@uni-wuppertal.de, Thorsten Benter; e-mail: tbenter@uni-wuppertal.de the low- to non-polar analyte regime. It was demonstrated earlier that selected compound classes, e.g., polycyclic aromatic hydrocarbons (PAH), are detectable two to three orders of magnitude more sensitive than with all other API methods, and a lower detection limit (LOD) in the amol regime has been achieved [2-4]. Furthermore, APLI is compatible with direct infusion stages, liquid chromatography (LC) [3], as well as with atmospheric pressure (AP) gas chromatography [5]. Along this line, rapid change from LC to GC operation, change of ionization methods (ESI, APCI, APLI), as well as true multi-mode operation (ESI/APLI) was 
recently demonstrated with a novel multi-purpose ion source (MPIS), coupled to a Bruker micrOTOF orthogonal time of flight (oaTOF) mass spectrometer (MS) [5].

In all cases involving APLI, bench top excimer lasers were used as radiation source. These systems are readily available and provide light power densities on the order of low MW/ $\mathrm{cm}^{2}$ without the need of optical focusing devices. The impressive improvement of excimer laser performance over the past decade is mainly driven by the application for medical (e.g., refractive laser eye surgery $[2,6]$ ) as well as in engineering (e.g., laser lithography $[7,8]$ ) purposes. Today, more than 400 million laser pulses can be fired without refurbishing of the laser tube [9]. Due to the modular design, all components requiring maintenance can be handled in-house. The gas lifetime has increased by orders of magnitude as well; modern excimer laser systems feature an automatic gas refill option once the laser energy drops below a user specified level. However, as mentioned by Short et al. [10], "APLI is not yet widely used due to the large size, expense and maintenance associated with the laser system."

We have recently begun to systematically investigate the properties of API sources, in particular with respect to improving the overall ion transmission efficiencies. Within this framework, spatially and temporally resolved measurements of the relative distribution of the sensitivity within the source enclosure (DIA [11-13]) have proven to be a very powerful tool. It was shown that the dynamic ion acceptance volume (DIAV), i.e., the volume element inside the source enclosure in which the sum of mass spectrometric signals assigned to one analyte does not drop below 1/e of the maximum value, is rather confined (see below). For a Bruker micrOTOF equipped with either the original Bruker APCI source or an MPIS, a typical two-dimensional projection of the DIAV is on the order of $40 \mathrm{~mm}^{2}$. Without additional optical devices the rectangular beam area of a table-top excimer laser is roughly $50 \mathrm{~mm}^{2}$ within the source region. It is speculated that laser radiation sources with considerable smaller beam diameters but delivering comparable power densities might generate comparable signal intensities if the beam path is carefully adjusted to overlap with the maximum of the corresponding DIAV. In this paper, a comprehensive discussion on the applicability of considerable smaller and more cost efficient UV-DPSS lasers in routine LC-APLI MS applications is presented.

\section{Experimental}

\section{Mass Spectrometer}

The mass spectrometric measurements were performed with a Bruker micrOTOF oaTOF MS (Bruker Daltonics, Bremen, Germany). The instrument was equipped with a home-built MPIS [5] featuring an identical APCI vaporization stage as used with the original Bruker source, cf. Figure 1. The continuous analyte liquid flow was delivered with a Hitachi L-7110 HPLC pump (Hitachi, Tokyo, Japan). Experimental

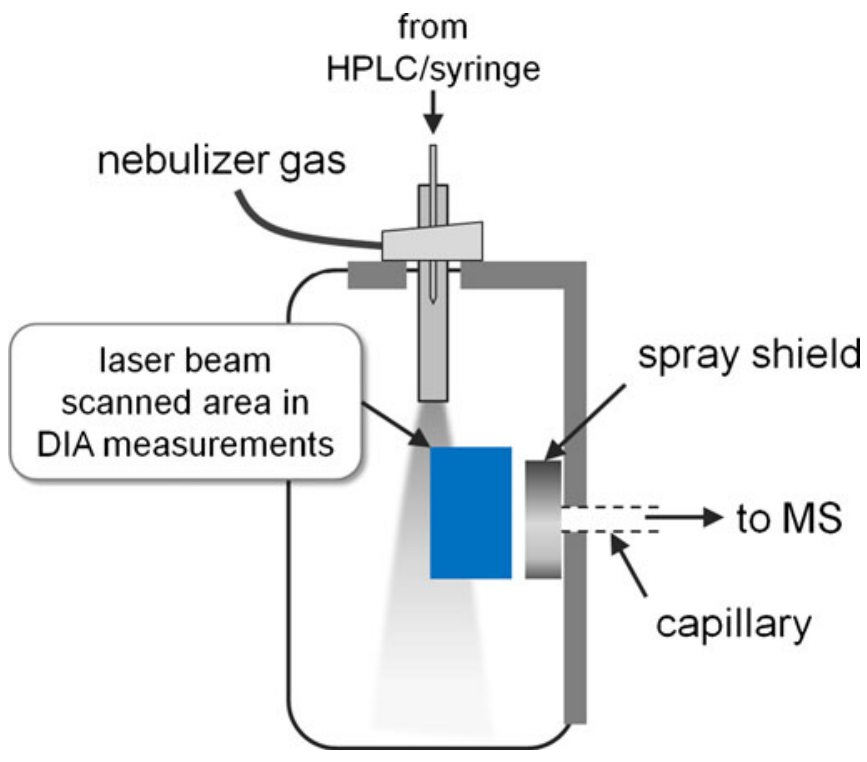

Figure 1. Schematic of the LC-APLI MS setup

settings for DIA investigations are given in the section "DIA measurements" further below.

The MS ion source parameter settings were as follows: Nebulizer gas pressure 3.0 bar, nebulizer temperature $320^{\circ} \mathrm{C}$, dry gas flow $3.0 \mathrm{~L} / \mathrm{min}$, dry gas temperature $200{ }^{\circ} \mathrm{C}$, spray shield voltage $-1000 \mathrm{~V}$, capillary voltage $-1000 \mathrm{~V}$. MS data were treated with the Bruker software package "DataAnalysis", ver. 3.4, including the calculation of $\mathrm{S} / \mathrm{N}$ ratios for individual mass signals. Three replicates were measured for each data point given (cf. Fig. 4).

The direct infusion solvent flow was $350 \mu \mathrm{L} / \mathrm{min}$ of a $10 \mathrm{nmol} / \mathrm{L}$ solution of pyrene in methanol (long range DIA contribution, see Results section), or $200 \mu \mathrm{L} / \mathrm{min}$ of a $0.9 \mu \mathrm{mol} / \mathrm{L}$ solution of pyrene in methanol (narrow range DIA contribution, see Results section).

\section{Lasers}

The excimer laser radiation source was an ATLEX 300 (ATL Lasertechnik, Wermelskirchen, Germany) system. The DPSS laser was a FQSS 266-50 (CryLas, Berlin, Germany). Figure 2 shows a scaled schematic of both systems.

The lasers were operated with an internally generated pulse frequency of $200 \mathrm{~Hz}$. Table 1 summarizes relevant laser beam data. It was shown earlier that synchronization of the laser pulse clock with the pulse sequence of the orthogonal acceleration stage of the TOF MS is not required when operating the ion source at atmospheric pressure [14].

\section{DIA Measurements}

Distribution of ion acceptance (DIA) measurements were performed as described in detail in previous publications $[12,13]$. Briefly, a $120 \mathrm{~mm}$ focal length quartz lens mounted on a computer controlled home-built $\mathrm{x}, \mathrm{y}$-translational stage 


\section{Excimer laser}

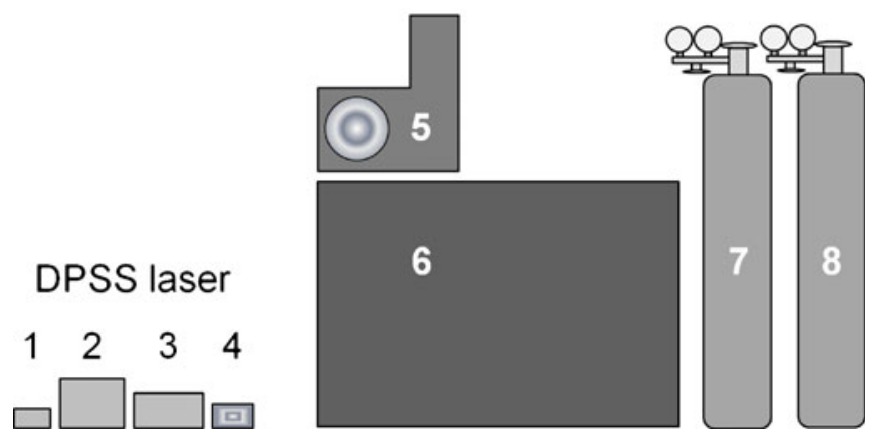

Figure 2. Scaled schematic of both laser systems used. Left: FQSS 266-50 DPSS laser; 1, external 24 V-power supply; 2, booster unit; 3 , control unit; 4 , laser head (may be directly attached to the source enclosure without any further optical devices). Right: ATLEX 300 excimer laser; 5, pump with halogen filter; 6, excimer laser unit (further optical devices required for directing the laser beam into the source enclosure); 7, compressed gas cylinder of the halogen/ krypton gas mixture, and 8, helium compressed gas cylinder, both for the operation of the excimer laser

was used to direct the focal point of the laser beam on a virtual grid inside the ion source enclosure. Ions are generated within a two-dimensional variable ionization volume of irregular shape along the laser beam propagation direction. DIA plots are projections of the spatially resolved sensitivity of the mass spectrometer on the $\mathrm{x}, \mathrm{y}$-plane spanned by the axes of the MS sampling port and the vaporization stage (for axis labeling, cf. Figures 3 and 4). The entire DIA data acquisition process is automated using home-built computer hard- and software components; a $15 \times$ $15 \mathrm{~mm}^{2}$ grid scan with a resolution of $1 \mathrm{~mm}$ in each direction is sufficient to register structural features in the DIA and is completed within $40 \mathrm{~min}$. Subsequent off-line data treatment yields plots as shown in Fig. 3. Pyrene as analyte is delivered as $1 \mu \mathrm{M}$ liquid solution in $\mathrm{MeOH}$ with liquid flows of $100 \mu \mathrm{L} / \mathrm{min}$. The common micrOTOF parameters for the DIA investigations presented in this work are as follows: $200{ }^{\circ} \mathrm{C}$ desolvation temperature, $320{ }^{\circ} \mathrm{C}$ vaporizer temperature, grounded spray shield, $180 \mathrm{~L} / \mathrm{h}$ desolvation gas flow, and 3 bar nebulizer pressure.

Table 1. Operational Parameters for the Laser Radiation Sources

\begin{tabular}{lll}
\hline & $\begin{array}{l}\text { ATLEX 300 } \\
\text { (excimer laser) }\end{array}$ & $\begin{array}{l}\text { FQSS 266-50 } \\
\text { (DPSS laser) }\end{array}$ \\
\cline { 2 - 3 } & $248 \mathrm{~nm}$ & $266 \mathrm{~nm}$ \\
Wavelength & $8 \mathrm{~mJ}$ & $60 \mu \mathrm{J}$ \\
Pulse energy & $200 \mathrm{~Hz}$ & $200 \mathrm{~Hz}$ \\
Repetition rate & $5 \mathrm{~ns}$ & $1 \mathrm{~ns}$ \\
Pulse width (FWHM) & Rectangular & Circular \\
Beam profile & $0.5 \mathrm{~cm}^{2}$ & $2 \times 10^{-3} \mathrm{~cm}^{2}$ \\
Beam area & $3 \times 10^{6} \mathrm{~W} \cdot \mathrm{cm}^{-2}$ & $3 \times 10^{7} \mathrm{~W} \cdot \mathrm{cm}^{-2}$ \\
Calc. power density & & \\
\hline
\end{tabular}

\section{Chemicals}

Methanol was obtained from Fisher Scientific, Waltham, MA, USA, pyrene was purchased from Merck KGaA, Darmstadt, Germany. Nitrogen from compressed gas cylinders with a stated purity of $99.9995 \%$ vol. (Gase.de, Sulzbach, Germany) was used as supply for the nebulizer and dry gas flows. All solvents were of analytical or chromatographic purity, all other chemicals of highest purity available, and were used without further purification.

\section{Results and Discussion}

\section{General DIA Investigations}

In common APLI applications, the laser beam is directed orthogonally to the MS entrance and illuminates a small volume in the ion source every $5 \mathrm{~ms}(200 \mathrm{~Hz})$. Due to the high gas throughput and the prevailing gas flow characteristics within the source enclosure, i.e., a confined gas stream extending from the vaporizer down to the source floor with average gas velocities in the propagation direction of up to $8 \mathrm{~m} / \mathrm{s}$ [15], it is estimated that a neutral analyte molecule is irradiated at most only once. In this case, the recorded mass spectrometric signal, with respect to ion source settings (gas flows, electrical potential gradients, and laser beam position), depends on two basically orthogonal parameters: (1) The spatial overlap of the neutral analyte density distribution with the laser beam irradiated volume (affecting the ionization efficiency), and (2) the overlap of the resulting ion population with the spatial distribution of ion acceptance of the MS (affecting the in-source detection efficiency). Both effects depend on the laser beam position and on gas flow dynamics; the in-source detection efficiency is furthermore influenced by electrical potential gradients. It follows that a DIA measurement depicts the product of the ionization and in-source detection efficiencies. In-depth investigations regarding DIA data were performed previously to characterize ion transmission efficiencies in API sources [11, 12, 1517]. The analysis of DIA plots suggests that a reasonable fraction of the achievable signal intensity may be covered utilizing much smaller laser beam cross sections than used in routine measurements with excimer lasers. The quantitative interpretation of DIA data is based on the assumption that the sensitivity distribution integrated over the illuminated volume is correlated with the recorded MS ion signal. The results of routine APLI experiments in combination with interpolated data from spatially resolved measurements allow estimating the change in sensitivity for a changing ionization volume: for a $0.5 \mathrm{~mm}$ diameter laser beam and a typical DIA data set, fractions of up-to $0.7 \%$ of the maximum signal achieved with a $7 \times 7 \mathrm{~mm}^{2}$ rectangular beam can be derived from such estimations. Neglecting the bottleneck nature of the transfer capillary entrance, any space charge and shielding effects, which both constrain the forces on ions toward the first pressure reduction stage, represent an underestimation of the expected sensitivity for 

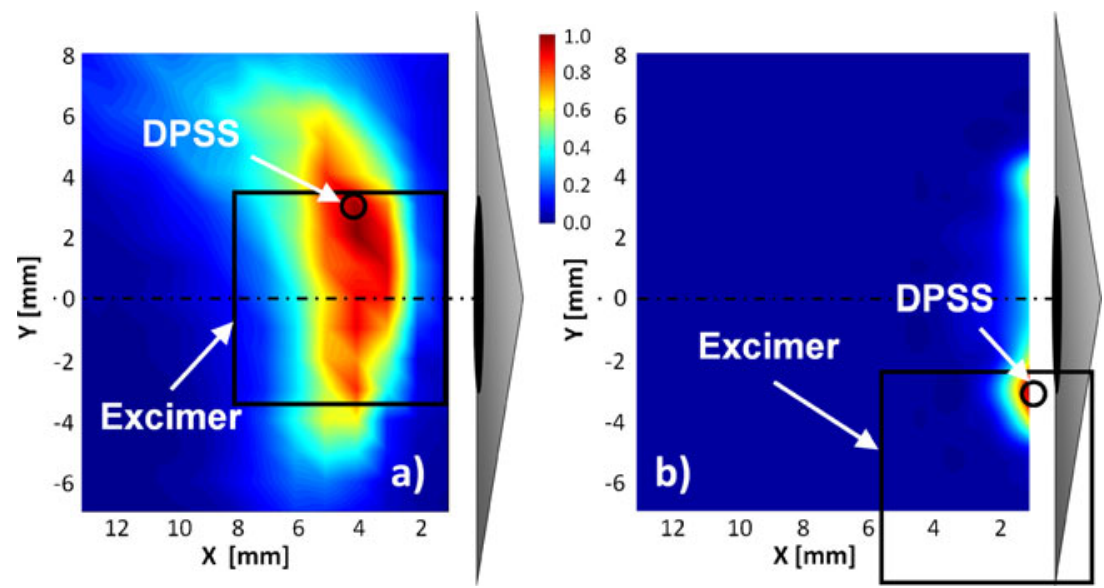

Figure 3. Signal-optimized laser positions mapped on significantly different DIA (cf. blue area in Fig. 1) plots. The spatial sensitivity distribution in AP ion sources results from the complex interaction of various ion source parameters; the spatial extent of the signal contributions has to be evaluated with respect to the limited ionization volume created by the ionizing light source. Signal intensities recorded upon irradiating a pyrene solution in $\mathrm{MeOH}$ at positions of maximum signal intensities obtained with the excimer laser (rectangle) and the DPSS laser (circles), respectively. For "long range" (panel a) ion source parameter settings, the excimer laser generated $1.6 \times 10^{6} \mathrm{cts}$, the DPSS laser $1.3 \times 10^{4} \mathrm{cts}$; for "narrow range" settings, the excimer laser generated $1.2 \times 10^{6} \mathrm{cts}$, the DPSS laser $1.4 \times 10^{5} \mathrm{cts}$. The color scale (top center) applies to both panels
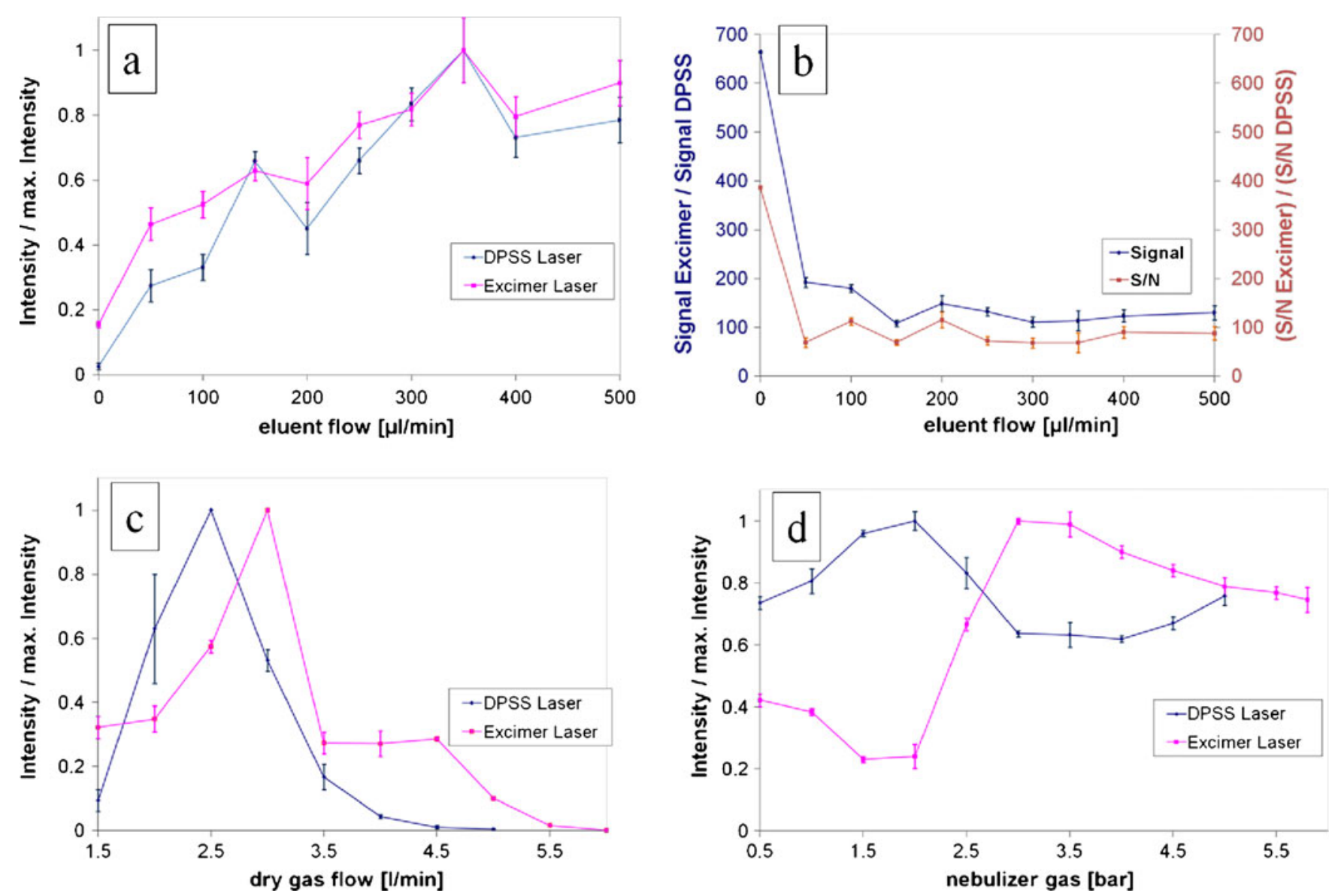

Figure 4. (a) Relative signal intensities of the DPSS and the excimer laser plotted as function of the eluent flow; (b) ratio of the DPSS/excimer laser signal plotted as function of the eluent flow; (c) relative signal intensities of the DPSS and the excimer laser plotted as function of the dry gas flow; (d) relative signal intensities of the DPSS and the excimer laser plotted as function of the nebulizer gas pressure. All error bars represent the standard deviation of three replicates 
the narrow laser beam. It is noted that differences in the power density of the two laser systems (DPSS: $3 \times 10^{7}$ $\mathrm{W} \cdot \mathrm{cm}^{-2}$; excimer: $3 \times 10^{6} \mathrm{~W} \cdot \mathrm{cm}^{-2}$ ), as well as differences in the absorption cross sections of the analyte pyrene between $248 \mathrm{~nm}$ and $266 \mathrm{~nm}\left(\sigma_{266 \mathrm{~nm}} / \sigma_{248 \mathrm{~nm}}=2.6\right.$ [18] $)$ are not considered in this calculation and further affirm the estimation as a lower limit. Hence, $0.7 \%$ is a rough estimate of the lower limit for the performance of a narrow beam area DPSS laser compared with a wide beam area excimer laser.

\section{DPSS laser vs. Excimer Laser in LC-APLI MS}

Dependence on Spatial Sensitivity Distribution (DIA data) Common ion source parameter settings for routine LC-APLI MS generate a considerable "large" DIAV. Such sensitivity distributions require strong electrical fields facing toward the transfer capillary and voltage settings of typically $-1000 \mathrm{~V}$ at the sampling orifice. The main signal contribution observed as a projection in two-dimensionally resolved experiments is generally of ellipsoidal shape with a vertical extension of $14 \mathrm{~mm}$, a horizontal extension of $3 \mathrm{~mm}$ and an area of $35 \mathrm{~mm}^{2}$ (cf. Fig. 3a). The DIAV extends up to a distance of about $3 \mathrm{~mm}$ to the spray shield and is nearly symmetrically arranged around the horizontal axis of the capillary entrance. Thorough manual scans with the unfocused excimer laser beam and the narrow beam of the DPSS laser were performed using a $0.01 \mu \mathrm{M}$ pyrene solution with direct syringe injection. As shown in Fig. 3a, the manually obtained signal-optimized positions of the excimer and the DPSS laser beams coincide very well with the observed maximum in the DIA. The recorded mass spectra for these ion source conditions reveal a relative signal intensity of $0.9 \%$ of the DPSS laser generated signal compared with the signal obtained with the excimer laser. This experimental result is very close to the above estimated lower limit of $0.7 \%$. The deviation is to be expected due to neglecting the impact of differing laser light power densities and the slightly higher absorption cross section for pyrene at $266 \mathrm{~nm}$ compared with $248 \mathrm{~nm}$ [18]. Of analytical importance is the gain in the $\mathrm{S} / \mathrm{N}$ ratio that is accompanied with the significant signal decrease choosing a smaller ionization volume. The $\mathrm{S} / \mathrm{N}$ ratio obtained with the DPSS laser accounts for $1.4 \%$ of the $\mathrm{S} / \mathrm{N}$ ratio achieved with the excimer laser. This is further associated with a 70 times lower detection limit obtained with the DPSS laser compared with the excimer laser. In addition, the increased $\mathrm{S} / \mathrm{N}$ performance in contrast to the relative signal intensities shows that the excimer laser generates a higher noise level than the DPSS laser, which is in full accord with the observations from DIA measurements: The narrow DPSS laser beam is preferably placed on a DIA position with significant signal contribution, whereas the wide $7 \times 7 \mathrm{~mm}^{2}$ area of the excimer laser beam typically exceeds the twodimensional projection of the DIAV (cf. Fig. 3).

In the case of ion source parameter settings leading to rather confined DIAV by, e.g., selecting small voltages on the sampling orifice, the obtained two-dimensional projection is represented by a small, rectangular "band" with a vertical extension of only $9 \mathrm{~mm}$, a horizontal extension of $2 \mathrm{~mm}$ $\left(18 \mathrm{~mm}^{2}\right)$. It is located nearly symmetrically around the horizontal axis of the capillary entrance and is almost directly "attached" to the spray shield (cf. Fig. 3b). The distance of the grid points closest to the spray shield in DIA data sets is typically chosen to be $1 \mathrm{~mm}$, whereas the manually scanned area with the DPSS laser well covers this artificial corridor. Hence, a quantitative comparison of theory and experiment is hardly possible in this case. The roughly extrapolated maximum in the recorded DIA, which is located directly at the spray shield entrance, is consistent with the maximum signal intensity obtained in the manual scan. Even the limited data set on the maximum achievable signal intensities for both laser systems (cf. Fig. 3, caption) illustrates a performance gain for the narrow laser beam light source with a decreasing spatial extent of DIA contributions due to changed ion source conditions: the data reveal an increased relative performance of $12 \%$ (DPSS versus excimer laser). The achievable spatially resolved signal intensity, however, is decreased by an estimated factor of 100 compared with the parameter settings typically chosen for routine LC-APLI MS operation utilizing excimer laser systems and DIA exhibiting large areas of significant contribution.

The experimental results are in good agreement with the quantitative estimate based on DIA data and demonstrate their value as a tool for routine LC-APLI MS. From an analytical point of view the results show that the application of a narrow-beam DPSS laser in routine LC-APLI MS with common API source enclosures (cf. Fig. 1) is inevitably leading to a loss of signal intensity by two orders-ofmagnitude, compared with the use of a wide-beam excimer laser. However, lower detection limits (LODs) of $10^{-1} \mathrm{fmol}$ for PAHs in conventional LC-APLI MS [19] relate to LODs for the DPSS laser application which are still in the lower pmol to upper fmol regime.

\section{Fluid Dynamical Impact on Signal Intensity}

The signal response to varying flow parameters was investigated for both laser systems with fixed beam positions and electrical potential settings, respectively, according to Fig. 4 and data given in the caption of Fig. 3 (DIA with "long-range" contribution).

\section{Impact of the Liquid Flow (Fig. 4a, b)}

Comparable signal responses to changes in the eluent flow are obtained for both laser systems (cf. Fig. 4a). The slopes noticeably reach a plateau at higher flow rates exceeding $300 \mu \mathrm{L} / \mathrm{min}$. Fluid dynamical models of this type of ion source design readily explain this behavior, as shown by Barnes et al. [20]. Here, the neutral analyte distribution within the source enclosure is proposed to reach an 
isotropically equilibrated state when operated in a continuous flow mode. It follows that above a certain continuously infused flow rate the dependency of the obtained MS signal switches from a mass flow to a concentration dependent behavior.

Plotting the ratio of the pyrene signal obtained with the excimer laser to the signal intensity obtained with the DPSS laser as function of the liquid flow results in an average factor of about 130 at flow rates of $150 \mu \mathrm{L} / \mathrm{min}$ up to $500 \mu \mathrm{L} /$ min (cf. Fig. 4b). Below $150 \mu \mathrm{L} / \mathrm{min}$ this ratio significantly increases up to 680 close to $0 \mu \mathrm{L} / \mathrm{min}$. The slope of the compared $\mathrm{S} / \mathrm{N}$ ratios obtained with the two laser systems (Fig. 4b) follows the signal intensity comparison plot with a negative intercept. The excimer laser $\mathrm{S} / \mathrm{N}$ performance is on average a factor of 90 better from 50 up to $400 \mu \mathrm{L} / \mathrm{min}$ eluent flow. Again, close to $0 \mu \mathrm{L} / \mathrm{min}$ the $\mathrm{S} / \mathrm{N}$ with the excimer laser dramatically increases, indicating a significantly higher sensitivity toward background analyte at low liquid flow rates. This result is consistent with investigations on the complex flow dynamics [15, 20], which suggest increasingly higher memory effects at low liquid flow rates to which a large laser beam is more sensitive.

\section{Impact of the Dry Gas Flow (Fig. 4c)}

The signal obtained with the DPSS laser rapidly drops below $10 \%$ of the maximum signal upon changes of $\pm 1 \mathrm{~L} /$ min dry gas flow. The excimer laser has a larger tolerance range towards variations of dry gas flows. This behavior was expected, since changes in ion source parameters typically result in spatial shifts of the patterns observed in the DIA $[11,12]$ that are well buffered by a wider laser irradiated area.

\section{Impact of Nebulizer Gas Pressure (Fig. 4d)}

Utilization of the excimer laser shows a higher sensitivity towards the nebulizer gas flow with a significant drop in intensity below an operational pressure of 2.5 bar whereas the DPSS laser generated signal is barely affected. The different behavior is readily explained by inspection of the spatial sensitivity distribution: DIA measurement series on the effect of ion source parameters frequently exhibit a strong change of the volume extension with significant contribution to the MS signal, whereas the maximum of the distribution is hardly affected. In this case the ionization volume extension determines the signal dependence on the parameter: A narrow laser beam confined to the virtually constant maximum location of the sensitivity distribution shows almost no dependence. In contrast, a large laser beam covering areas with a significantly changed contribution reflects these changes in a distinct dependence. The ion source parameter "nebulizer gas" provides an example for such behavior $[11,17,21]$.
The different behavior of both laser systems toward changes of ion source parameters is readily explained in terms of the spatial sensitivity distribution in the AP ion source, as demonstrated here for the dependence on dry gas and nebulizer gas flows, respectively. Altering the dry gas flow significantly shifts the maximum of the DIA. Consequently, narrow laser beam systems show a lower tolerance towards changes of ion source parameters whereas the wide excimer laser beam very well buffers such fluctuations. Furthermore, changes in the spatial extent of the maximum in the DIA, as obtained upon nebulizer gas pressure variations, barely affect the DPSS laser generated signal, whereas the excimer laser generated signal exhibits a significant drop in intensity with decreasing pressure below 2.5 bar. The ultimate goal of adjusting the ion source parameters is to maximize the overlap of the ion acceptance distribution and the ionization volume with the long-range distribution of analyte ions in the AP ion source. One approach to meet this demand is a restriction of the analyte "spread" to a closely confined volume, which would then potentially become to its whole extent the ionization volume.

\section{Summary and Conclusion}

DIA measurements have been applied to qualitatively and quantitatively predict the effect of laser light source selection in routine LC-APLI MS application. A theoretical lower limit of $0.7 \%$ for the relative performance of a small beam area $\left(2.0 \times 10^{-3} \mathrm{~cm}^{2}\right)$ compared with a wide beam of $0.5 \mathrm{~cm}^{2}$ as estimated on the basis of DIA data treatment is supported by the experimental data of $0.9 \%$ accompanied by a significant reduction (1.4\%) of the $\mathrm{S} / \mathrm{N}$ ratio in comparison to the wide-beam excimer laser application.

Investigations on varying flows (eluent, dry gas, nebulizer gas flow) reveal a comparable behavior for both laser systems for typical conditions, whereas a significant performance gain of the DPSS laser application could be observed under virtually "non-liquid-flow" conditions. These observations of a higher sensitivity toward background analyte contributions utilizing a wide ionization volume, as well as the non-linear dependence on the eluent flow are consistent with our current investigations on the turbulent and complex flow dynamics within the ion source $[15,20]$.

For the applicability of DPSS lasers in common LC-APLI MS applications we conclude: (1)With LODs in the $10^{-1}$ fmol regime when using excimer lasers [19], the DPSS utilization resulting in signal intensities of $0.9 \%$ is applicable for routine LC-APLI MS when LODs for PAHs of lower pmol to fmol are sufficient, particularly with a significantly reduced lower detection limit. (2) The DPSS laser system is more sensitive to selected ion source parameters that cause a spatial shift in the DIA maximum (dry gas flow and high voltage settings), but (3) the DPSS laser is less sensitive toward other parameters that affect the spatial extent of the analyte signal 
contribution in DIA measurements (e.g., the pressure of the nebulizer gas). (4)The maximum ionization energy for (1+1)-REMPI drops from $10 \mathrm{eV}(248 \mathrm{~nm})$ to $9.3 \mathrm{eV}$ $(266 \mathrm{~nm})$. (5) The size, acoustic noise level (DPSS: around $0 \mathrm{~dB}$; excimer: $70 \mathrm{~dB}$ [9]), maintenance and purchase cost (DPSS: $\sim 10 \mathrm{k} €$; Excimer: $\sim 40 \mathrm{k} €$ ) of the DPSS laser significantly outperforms the excimer laser in terms of handling and cost efficiency. For the analytical application of the APLI method utilizing the presently employed sources both laser systems have their specific advantages and the choice of the preferred light source is strongly application driven. A similar comparison of DPSS laser versus excimer laser performance for routine GC-APLI MS is under investigation.

The potential of a significant performance gain of narrow-beam laser light source by spatially fitting the laser irradiated volume and the analyte distribution provides the motivation for current efforts to design novel API sources. In the near future, the coaxial concept of directing the ionizing laser beam through a laminar flow of bulk gas containing the analyte [20] will establish an LC-APLI MS method that is capable of using all the benefits of the small DPSS lasers with a comparable performance currently obtained with an excimer laser. This design, along with advantages and restrictions, will be discussed in depth in an upcoming publication.

\section{Acknowledgments}

The authors acknowledge financial support for this work by the German Research Foundation (DFG) under contracts BE 2124/6-1 and BE 2124/4-1. Generous support of Bruker Daltonics, Bremen, Germany, is gratefully acknowledged.

\section{References}

1. Commercial availability of APLI-sources. http://www.bdal.com/products/ lc-ms/apli-source/overview.html

2. Lorenz, M.; Short, L. C.; Constapel, M.; Schellenträger, M.; Möschter, S.; Schmitz, O. J.; Gäb, S.; Brockmann, K.-J.; Droste, S.; Benter, T. Atmospheric Pressure Laser Ionization (APLI) Mass Spectrometry for Measurement of Aromatic Hydrocarbons with Low Polarity. Proceedings of the 53rd ASMS Conference on Mass Spectrometry and Allied Topics; San Antonio, TX, June 2005.

3. Constapel, M., Schellenträger, M., Schmitz, O.J., Gäb, S., Brockmann, K.J., Giese, R., Th, B.: Atmospheric-Pressure Laser Ionization: A Novel Ionization Method for Liquid Chromatography/Mass Spectrometry. Rapid Commun. Mass Spectrom. 19(3), 326-336 (2005)

4. Schiewek, R., Schellenträger, M., Mönnikes, R., Lorenz, M., Giese, R., Brockmann, K.J., Gäb, S., Benter, T., Schmitz, O.J.: Ultrasensitive Determination of Polycyclic Aromatic Compounds with AtmosphericPressure Laser Ionization as an Interface for GC/MS. Anal. Chem. 79 (11), 4135-4140 (2007)
5. Schiewek, R., Lorenz, M., Giese, R., Brockmann, K., Benter, T., Gäb, S., Schmitz, O.: Development of a Multipurpose Ion Source for LC-MS and GC-API MS. Anal. Bioanal. Chem. 392(1), 87-96 (2008)

6. McDonald, M.B., Kaufman, H.E., Frantz, J.M., Shofner, S., Salmeron, B., Klyce, S.D.: Excimer Laser Ablation Human Eye. Arch. Ophthalmol. 107(5), 641-642 (1989)

7. Solomon, K.D., Fernández de Castro, L.E., Sandoval, H.P., Biber, J.M., Groat, B., Neff, K.D., Ying, M.S., French, J.W., Donnenfeld, E.D., Lindstrom, R.L.: LASIK World Literature Review: Quality of Life and Patient Satisfaction. Ophthalmology 116(4), 691-701 (2009)

8. Levinson, H. J. Principles of Lithography. SPIE - The International Society for Optical Engineering, 2nd ed.; Bellingham, 2005.

9. ATL Lasertechnik GmbH; User Manual Excimer Laser ATLEX-SI, 2008.

10. Short, L.C., Cai, S.S., Syage, J.A.: APPI-MS: Effects of Mobile Phases and VUV Lamps on the Detection of PAH Compounds. J. Am. Soc. Mass Spectrom. 18(4), 589-599 (2007)

11. Lorenz, M.: Räumlich und zeitlich aufgelöste Photoionisierung als Werkzeug zur Charakterisierung von Atmosphärendruckionenquellen in der Massenspektrometrie; Dissertation. University of Wuppertal, Germany (2010)

12. Lorenz, M., Schiewek, R., Brockmann, K.J., Schmitz, O.J., Gäb, S., Benter, T.: The Distribution of Ion Acceptance in Atmospheric Pressure Ion Sources: Spatially Resolved APLI Measurements. J. Am. Soc. Mass Spectrom. 19(3), 400-410 (2008)

13. Wissdorf, W.; Lorenz, M.; Brockmann, K. J.; Schmitz, O. J.; Gäb, S.; Benter, T. Determination of the Distribution of Ion Acceptance (DIA) of Atmospheric Pressure Ionization Sources. Proceedings of the 54th ASMS Conference on Mass Spectrometry and Allied Topics; Indianapolis, IN, June 2007.

14. Lorenz, M.; Klee, S.; Mönnikes, R.; Mangas Suarez, A. L.; Brockmann, K. J.; Schmitz, O. J.; Gäb, S.; Benter, T. Atmospheric Pressure Laser Ionization (APLI): Investigations on Ion Transport in Atmospheric Pressure Ion Sources. Proceedings of the 56th ASMS Conference on Mass Spectrometry and Allied Topics; Denver, CO, June 2008.

15. Wissdorf, W.; Pohler, L.; Pöhler, T.; Hönen, H.; Brockmann, K. J.; Benter, T. Modular Computational Toolset for Atmospheric Pressure Ionization Method Development: SIMION meets FEM. Proceedings of the 58th ASMS Conference on Mass Spectrometry and Allied Topics; Salt Lake City, UT, May 2010.

16. Lorenz, M.; Wissdorf, W.; Klee, S.; Kersten, H.; Brockmann, K. J.; Benter, T. Ion Transport Processes in API Sources: Temporally and Spatially Resolved APLI Measurements. Proceedings of the 57th ASMS Conference on Mass Spectrometry and Allied Topics; Philadelphia, PA, June 2009.

17. Lorenz, M.; Wissdorf, W.; Klee, S.; Kersten, H.; Brockmann, K. J.; Benter, T. Spatially and Temporally Resolved Atmospheric Pressure Laser Ionization as a Powerful Tool for the Characterization of Ion Sources: An overview. Proceedings of the 58th ASMS Conference on Mass Spectrometry and Allied Topics; Salt Lake City, UT, May 2010 .

18. Thony, A., Rossi, M.J.: Gas-Phase UV Spectroscopy of Anthracene, Xanthone, Pyrene, 1-Bromopyrene, and 1,2,4-Trichlorobenzene at Elevated Temperatures. J. Photochem. Photobiol. A Chemistry 104(1/ 3), 25-33 (1997)

19. Schiewek, R.: Entwicklung einer Multi-Purpose Ionenquelle für die APMS sowie Design und Anwendung von APLI-Ionisationslabeln; Dissertation. University of Wuppertal, Wuppertal (2008)

20. Barnes, I.; Kersten, H.; Wissdorf, W.; Pöhler, T.; Hönen, H.; Klee, S.; Brockmann, K. J.; Benter, T. Novel Laminar Flow Ion Sources for LCand GC-API MS. Proceedings of the 58th ASMS Conference on Mass Spectrometry and Allied Topics; Salt Lake City, UT, May 2010.

21. Lorenz, M., Wißdorf, W.; Brockmann, K. J.; Benter, T., unpublished (paper in preparation to be submitted to JASMS). 